\title{
Microtexture of magnetite thin films of (001) and (111) orientations on MgO substrates studied by electron-backscatter diffraction
}

\author{
A. Koblischka-Veneva ${ }^{a)}$ \\ Institute of Functional Materials, Saarland University, P.O. Box 151150, D-66041 Saarbrücken, Germany \\ M. R. Koblischka \\ Institute of Experimental Physics, Saarland University, P.O. Box 151150, D-66041 Saarbrücken, Germany \\ S. Murphy and S. K. Arora \\ SFI Nanoscience Laboratory, Trinity College, Dublin, Dublin 2, Ireland \\ F. Mücklich \\ Institute of Functional Materials, Saarland University, P.O. Box 151150, D-66041 Saarbrücken, Germany \\ U. Hartmann \\ Institute of Experimental Physics, Saarland University, P.O. Box 151150, D-66041 Saarbrücken, Germany \\ I. V. Shvets \\ SFI Nanoscience Laboratory, Trinity College, Dublin, Dublin 2, Ireland
}

(Presented on 6 November 2007; received 1 October 2007; accepted 9 November 2007; published online 13 February 2008)

The grain orientation of (001)- and (111)-oriented magnetite thin films grown on $\mathrm{MgO}$ substrates (film thickness of $100-400 \mathrm{~nm}$ ) is analyzed by means of the electron-backscatter diffraction (EBSD) technique. The (001) surface after a short annealing in air $\left(1 \mathrm{~min}, 250{ }^{\circ} \mathrm{C}\right)$ is characterized by the presence of tiny (diameter of 100-200 nm) misoriented islands, which have an influence on the antiferromagnetic coupling within the film. In the (111)-oriented films, such defects are found to be absent, and the films show a very homogeneous surface. The achieved spatial resolution enables further a cross-section analysis of a 400-nm-thick film with (001) orientation, even close to the interface MgO-magnetite. (C) 2008 American Institute of Physics. [DOI: 10.1063/1.2837283]

\section{INTRODUCTION}

Magnetite $\left(\mathrm{Fe}_{3} \mathrm{O}_{4}\right)$ as a half-metallic ferromagnet is an interesting system for various applications because of its unusual magnetic properties for electron spin-manipulating devices. ${ }^{1,2}$ The high Curie temperature $\left(T_{C}=860 \mathrm{~K}\right)$ enables such devices to be operated at room temperature. However, the resulting magnetic properties of magnetite thin films grown on $\mathrm{MgO}$ substrates were found to be pretty peculiar, ${ }^{3-6}$ so a detailed investigation of the microstructure of these films is required. Using the electron-backscatter diffraction (EBSD) technique, we could already analyze the crystallographic orientation of (001)-oriented magnetite films and identify the typical defects within the sample surface. ${ }^{7,8}$ These defects can play an important role as pinning sites as observed in magnetic force microscopy (MFM) experiments. ${ }^{9}$ In this contribution, we extend these investigations to (111)-oriented magnetite films and the cross sections of (001)-oriented magnetite films.

\section{EXPERIMENTAL DETAILS}

Epitaxial magnetite thin films with a thickness of $100-400 \mathrm{~nm}$ were grown on $\mathrm{MgO}$ single-crystal substrates cut along the [100] and [111] directions within $\pm 0.1^{\circ}$ by

\footnotetext{
${ }^{a)}$ Electronic mail: a.koblischka-veneva@mx.uni-saarland.de.
}

oxygen-plasma-assisted molecular beam epitaxy. Details about the preparation and the in situ reflection high energy electron diffraction analysis can be found in Ref. 10. The film thickness was controlled by quartz-crystal thickness monitors calibrated using x-ray reflectivity. The samples were subsequently annealed in air at $250{ }^{\circ} \mathrm{C}$; for the present study, the duration of annealing was chosen to be $1 \mathrm{~min}$. For the EBSD investigation of cross sections, the magnetite films were embedded in electrically conducting resin containing $\mathrm{Cu}$ particles and were subsequently mechanically polished using diamond paste and a final step using colloidal silica (Struers OP-S).

The EBSD system employed here consists of a dual beam workstation (FEI Strata DB 235) equipped with a TSL OIM analysis unit. ${ }^{11}$ The Kikuchi patterns are generated at an acceleration voltage of $20 \mathrm{kV}$ and are recorded by means of a DigiView camera system. The working distance is set at $10 \mathrm{~mm}$. To produce a crystallographic orientation map, the electron beam is scanned over a selected surface area and the resulting Kikuchi patterns are indexed and analyzed automatically (i.e., the Kikuchi bands are detected by means of the software). An image quality (IQ) parameter and a confidence index (CI) is recorded for each such Kikuchi pattern. Based on the analysis of the recorded CI value, a multiphase analysis is realized. A detailed description of the measurement procedure can be found in Refs. 12 and 13; the Kikuchi patterns of magnetite are shown in Ref. 9. 

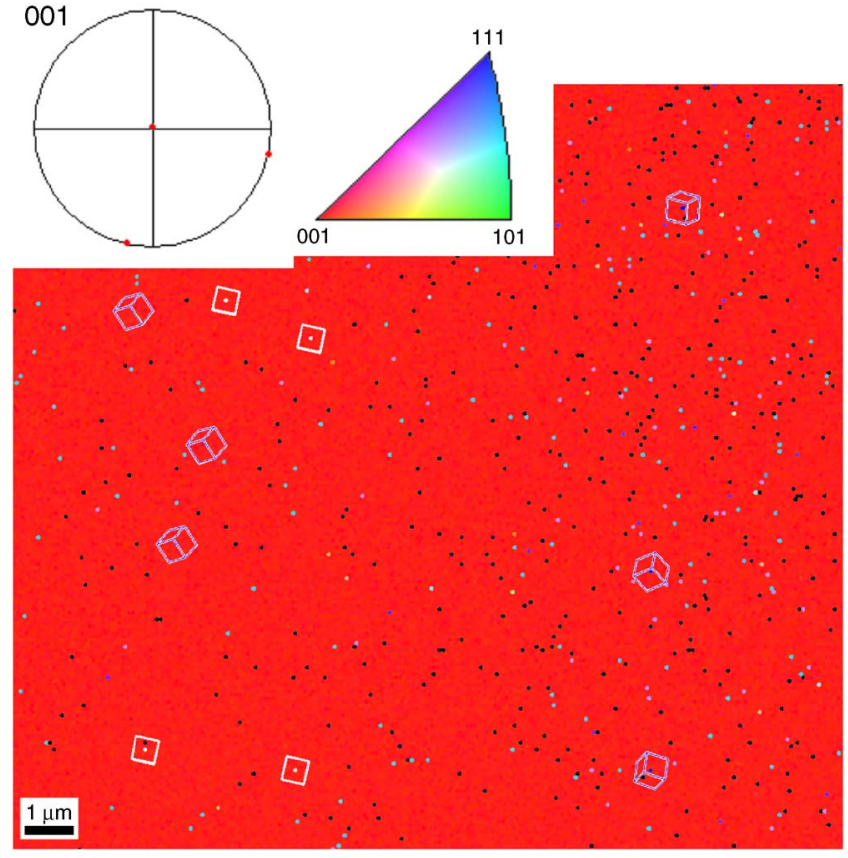

FIG. 1. (Color online) High-resolution IPF map of (001)-oriented magnetite (EBSD step size of $40 \mathrm{~nm}$ ). Additionally, the respective unit cells are plotted in order to visualize the misfits created by the misorientations detected. The black dots are voids or nonindexed points. The pole figure (inset) shows the locations of the drawn crystallographic orientations; the color code for the IPF map is given in the stereographic triangle. The scale bar is $1 \mu \mathrm{m}$ long.

\section{RESULTS AND DISCUSSION}

Figure 1 gives an inverse pole figure (IPF) map with a high EBSD resolution measured in the [001] direction of a (001)-oriented magnetite film. Additionally, we plot the respective unit cells in order to visualize the misfits created by the misorientations detected. In fact, the existing misoriented islands present on the surface have two main orientations (blue unit cells) as compared to the magnetite matrix (white unit cells). The misorientations found via the EBSD technique are in strong contrast to the highly oriented crystalline nature and layer-by-layer growth observed using highresolution multicrystal diffraction and RHEED techniques, respectively. ${ }^{14}$ The pole figure (inset) indicates the locations of the drawn orientations. These unit cell wire frames also indicate that two fully different faces of the magnetite unit cell come close to each other, which may have important consequences for the antiferromagnetic coupling between them. MFM measurements ${ }^{9,14}$ on the same samples have shown the presence of pinning sites, which exhibit similar size and distribution as these misoriented islands. The resulting consequences of this observation will have to be worked out in detail using modeling procedures.

In order to illustrate the nature of these misoriented islands, we further investigated magnetite samples with (111) orientation. Figure 2(a) presents an IQ map, resembling a backscattered electron image but being taken in EBSD configuration $\left(70^{\circ}\right.$ angle to the electron beam). Here, several scratches in the substrate are visible, which cause in turn misorientations within the magnetite. However, the areas in between are quite homogeneous. In Fig. 2(b) an IPF map in the [001] direction is shown, indicating the dominating (111)

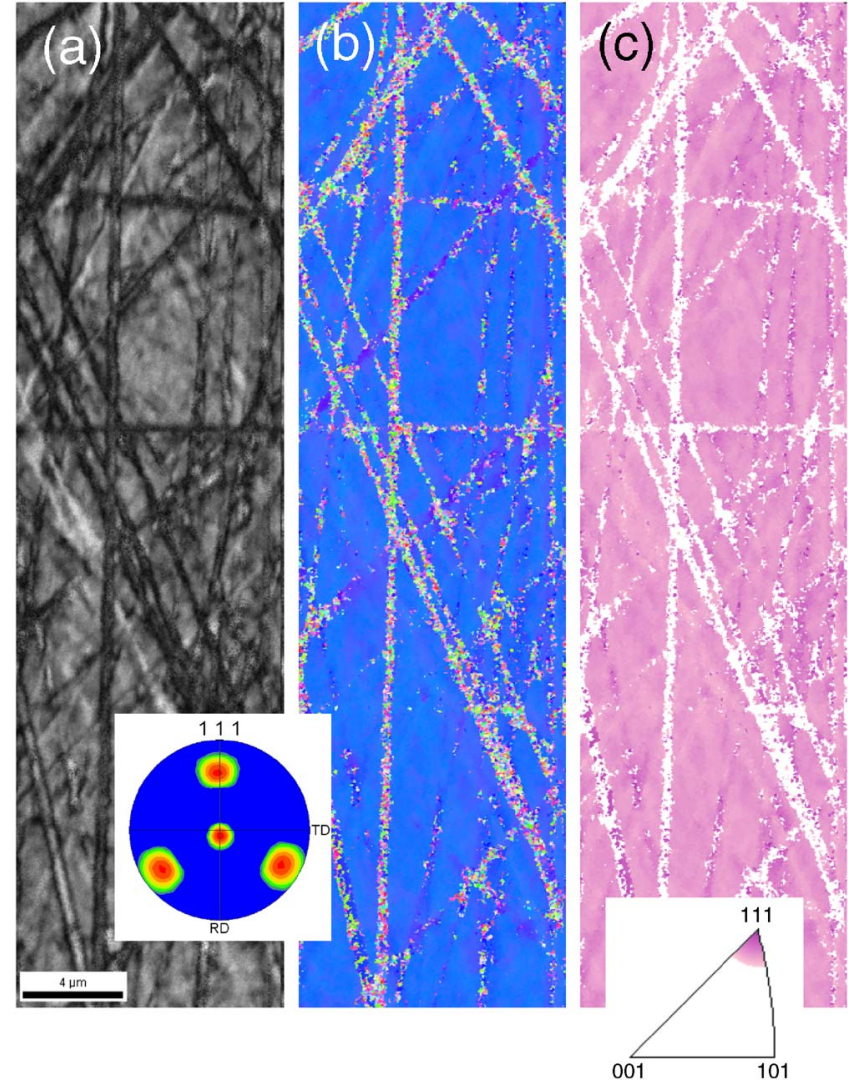

FIG. 2. (Color online) EBSD analysis of (111)-oriented magnetite. The left map (a) is an IQ map and the middle one (b) an IPF map in the [001] direction, giving the crystallographic orientation. The last map (c) is a CD map, together with the corresponding color code (triangle). The inset shows the EBSD-determined pole figure in the [111] direction. The length of the marker is $4 \mu \mathrm{m}$.

orientation. Along the defects stemming from the substrate, high-angle misorientations are created. Finally, in Fig. 2(c), the crystal direction (CD) map is given. Here, the colors are assigned close to the main poles, (001), (101), and (111), respectively, with a tolerance of $10^{\circ}$. This enables to visualize misorientations within one domain much clearer as compared to the IPF maps. The orientation of the magnetite is about $3^{\circ}-5^{\circ}$ off the (111) direction, similar to the earlier observations on the (001) surface. However, we have not found in this orientation the characteristic misoriented islands as in the (001) orientation. This will be important for further magnetic domain investigations using MFM.

Figure 3 presents a cross-section EBSD measurement in the form of a CD map. The most important region for our analysis around the (001) direction is chosen to have the full color scale. Therefore, a dark blue color would indicate a perfect (001) orientation, i.e., $0^{\circ}$ misorientation. The (101) direction is given by a color scale ranging between violet and black, and the (111) direction is visualized on a color scale between light blue and black for distinction. The MgO substrate (nonindexed points) is represented in black because due to charging effects, no analysis of the substrate was possible. As the main color of the map is light green and yellow, we see that there is an $\sim 3^{\circ}-5^{\circ}$ deviation from the optimum (001) direction throughout the magnetite film. Some (101)oriented grains are located close to the substrate surface, 


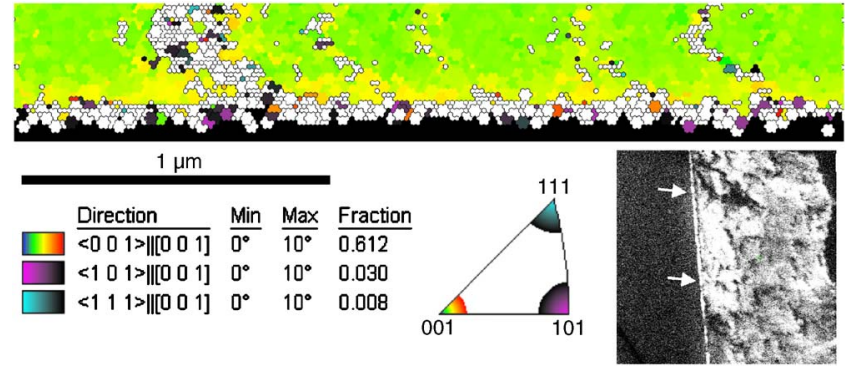

FIG. 3. (Color online) CD map for the cross section. The colors are given in a $10^{\circ}$ range around the main poles as indicated in the triangle below the map. Around the (001) direction, the full color scale is chosen in order to visualize the differences in the crystal growth. The inset shows a SEM image (in EBSD configuration) of the thin film cross section (arrows).

while the other misoriented grains do not have orientations close to one of the poles and are, therefore, colored in white. Close to the $\mathrm{MgO}$ surface, the yellow color $\left(\sim 5^{\circ}\right.$ misorientation) of the magnetite prevails, while toward the sample surface, this misorientation gradually changes toward green $\left(\sim 3^{\circ}\right.$ misorientation). The CD map further shows that even the green areas are not homogeneously distributed, but still some slightly misoriented subgrains do exist, similar to the case of the (111)-oriented film. The fractions of each detected orientation are also given below Fig. 3, with the dominating amount $(61 \%)$ being oriented close to the (001) direction. Again, the results obtained on cross-section samples using the EBSD technique do not agree with our earlier results of high-resolution TEM studies performed on (001) oriented $\mathrm{Fe}_{3} \mathrm{O}_{4}-\mathrm{MgO}$ hetero-interface ${ }^{14}$ and suggest that the charging effects may influence the image contrast and hence, the assignment of domain orientation.

The present EBSD analysis implies that the currently employed process of preparation of magnetite films leads to the formation of magnetite films which are oriented close to the respective pole direction, (001) and (111). The misorientation boundaries detected will improve upon oxygen annealing which cause a change in stoichiometry as shown in Refs.
7 and 8. The EBSD cross section analysis will be an important tool for future investigations if the current contradictions to the RHEED and TEM measurements can be solved. Furthermore, in our case in the (001)-oriented films, the misorientation obtained at the substrate may prevail in some locations in the film and is in this way responsible for the formation of the characteristic misoriented islands, which do not exist in the (111)-oriented magnetite film. This observation may have impact on the formation and pinning of magnetic domains in these films.

\section{ACKNOWLEDGMENTS}

This work is supported by DFG Project No. MU959/19 and the EU-funded project "ASPRINT" (Contract No. NMP4-CT-2003-1601), which is gratefully acknowledged.

${ }^{1}$ S. A. Wolf, D. A. Awschalom, R. A. Buhrman, J. M. Daughton, S. von Molnar, M. L. Roukes, A. Y. Chtchelkanova, and D. M. Treger, Science 294, 1488 (2001).

${ }^{2}$ G. A. Prinz, Science 282, 1660 (1998).

${ }^{3}$ D. T. Margulies, F. T. Parker, M. L. Rudee, F. E. Spada, J. N. Chapman, P. R. Aitchison, and A. E. Berkowitz, Phys. Rev. Lett. 79, 5162 (1997).

${ }^{4}$ D. T. Margulies, F. T. Parker, F. E. Spada, R. S. Goldman, J. Li, R. Sinclair, and A. E. Berkowitz, Phys. Rev. B 53, 9175 (1996).

${ }^{5}$ M. Ziese and H. J. Blythe, J. Phys.: Condens. Matter 12, 13 (2000).

${ }^{6}$ W. L. Zhou, K.-Y. Wang, C. J. O'Conner, and J. Tang, J. Appl. Phys. 89, 7398 (2001)

${ }^{7}$ A. Koblischka-Veneva, M. R. Koblischka, F. Mücklich, S. Murphy, Y. Zhou, and I. V. Shvets, IEEE Trans. Magn. 42, 2873 (2006).

${ }^{8}$ A. Koblischka-Veneva, M. R. Koblischka, Y. Zhou, S. Murphy, F. Mücklich, U. Hartmann, and I. V. Shvets, J. Magn. Magn. Mater. 316, e663 (2007).

${ }^{9}$ A. Koblischka-Veneva, M. R. Koblischka, J. D. Wei, Y. Zhou, S. Murphy, F. Mücklich, U. Hartmann, and I. V. Shvets, J. Appl. Phys. 101, 09M507 (2007).

${ }^{10}$ Y. Zhou, X. Jin, and I. V. Shvets, J. Magn. Magn. Mater. 286, 346 (2005).

${ }^{11}$ Orientation imaging microscopy software, Version V4.0, user manual, TexSEM Laboratories (TSL), Draper, UT, 2004.

${ }^{12}$ M. R. Koblischka and A. Koblischka-Veneva, Physica C 392-396, 545 (2003).

${ }^{13}$ A. Koblischka-Veneva, M. R. Koblischka, F. Mücklich, N. Hari Babu, and D. A. Cardwell, Physica C 426-431, 618 (2005).

${ }^{14}$ J. D. Wei, I. Knittel, Y. Zhou, S. Murphy, F. T. Parker, I. V. Shvets, and U. Hartmann, Appl. Phys. Lett. 89, 122517 (2006). 